\title{
Uso do Arduino para Ensino de Conceitos Intermediários de Programação
}

\author{
Rachel C. D. Reis ${ }^{1}$, Larissa F. Rodrigues ${ }^{1}$, Pablo L. A. Munhoz ${ }^{1}$, Kamila T. Lyra $^{2}$, \\ Seiji Isotani ${ }^{2}$ \\ ${ }^{1}$ Universidade Federal de Viçosa, Campus Rio Paranaíba \\ ${ }^{2}$ Universidade de São Paulo, Campus São Carlos \\ rachel.reiseufv.br, larissa.f.rodrigueseufv.br, pablo.munhoz@ufv.br, \\ kalyra_03@usp.br, sisotani@icmc.usp.br
}

\begin{abstract}
The use of Arduino to introduce basic programming concepts has been widely investigated by several studies in the literature. Despite of important contributions, we observed the lack of studies that evaluate such instrument to teach programming intermediate concepts, i.e., after the introductory programming topics. Therefore, this paper aims at comparing the influence of Arduino on students' motivation when used to solve basic and intermediate practical programming exercises. An experimental study carried out with 62 students showed that students felt less competent when using Arduino to study programming intermediate concepts.
\end{abstract}

Resumo. O uso do Arduino para introdução de conceitos iniciais de programação tem sido amplamente investigado por trabalhos na literatura. Apesar das importantes contribuições, observou-se a carência de estudos que avaliem esse instrumento para o ensino de conceitos intermediários, ou seja, posteriores aos tópicos introdutórios de programação. Logo, este artigo tem como objetivo comparar a influência do Arduino na motivação dos alunos quando utilizado na resolução de exercícios práticos básicos e intermediários de programação. Um estudo experimental realizado com 62 alunos mostrou que os alunos se sentiram menos competentes a utilizar o Arduino para estudo de conceitos intermediários de programação.

\section{Introdução}

Estudos mostram que o alto índice de evasão e reprovação nos cursos superiores da área de Computação está relacionado à dificuldade dos alunos em disciplinas iniciais do curso, por exemplo, de introdução à programação [Bosse e Gerosa 2015]. Nesse sentido, Zanetti et al. (2016) e Queiroz e Rebouças (2018) discutem o esforço da comunidade científica em desenvolver práticas pedagógicas e técnicas de ensino-aprendizagem (diferentes das convencionais) para amenizar esse cenário. Dentre essas práticas destaca-se o uso do Arduino.

O Arduino é uma placa eletrônica composta por circuitos com entradas e saídas para um microprocessador AVR que pode facilmente ser conectada a um computador via entrada USB e programada por meio de uma interface de desenvolvimento [Banzi e 
VIII Congresso Brasileiro de Informática na Educação (CBIE 2019)

Anais dos Workshops do VIII Congresso Brasileiro de Informática na Educação (WCBIE 2019)

Evans 2014]. Inclui hardware e software de código aberto com o intuito de oferecer versatilidade, fácil utilização e baixo custo, sendo amplamente utilizado em projetos na área de ensino [Fernandes et al. 2018, Silva et al. 2016].

Diferentes modelos de placas Arduino encontram-se disponíveis no mercado que podem ser vendidas isoladas ou em kits. Além do Arduino, os kits são formados por diversos componentes (ex.: LEDs, resistências, sensores e motores) que são acoplados em uma placa de ensaio (protoboard) e controlados por aplicações computacionais [Banzi e Michael 2014].

$\mathrm{Na}$ área da Computação, trabalhos na literatura [Cardoso e Antonello 2015, Rubio et al. 2015] têm utilizado o Arduino como um aliado no desenvolvimento de habilidades, atitudes e competências desejáveis no aprendizado de programação [Henrique e Tedesco 2017]. Por exemplo, desenvolvimento do raciocínio lógico, capacidade de abstração, construção do conhecimento, motivação pessoal, dentre outros.

Grande parte das pesquisas sobre o uso do Arduino no contexto da computação têm focado no ensino de conceitos básicos de programação [Fernandes et al. 2018, Zanetti e Oliveira 2015, Cardoso e Antonello 2015, Rubio et al. 2015]. Logo, este artigo tem como objetivo apresentar os resultados de um estudo experimental que compara a influência do Arduino na motivação dos alunos ao trabalhar os conceitos básicos e intermediários $^{1}$ de uma linguagem de programação.

Além dessa seção introdutória, este trabalho está organizado da seguinte forma: na Seção 2 são apresentados os trabalhos relacionados. Na sequência, a Seção 3 apresenta o método utilizado neste estudo. Na Seção 4 é apresentado o planejamento e desenvolvimento do estudo experimental. Na Seção 5 são apresentados e discutidos os resultados e, finalmente, na Seção 6 são apresentadas as conclusões.

\section{Trabalhos Relacionados}

As pesquisas na literatura sobre o uso do Arduino para o desenvolvimento do raciocínio lógico e ensino de conceitos de programação são aplicadas a alunos em diferentes níveis de escolaridade. Por exemplo, ensino fundamental [Fernandes et al. 2018], ensino médio-técnico [Zanetti e Oliveira 2015] e ensino superior [Cardoso e Antonello 2015, Rubio et al. 2015].

Fernandes et al. (2018) realizaram um estudo, em cinco encontros, com alunos do ensino fundamental para desenvolvimento da lógica de programação, a partir da prática de conceitos básicos de algoritmos: sintaxe, semântica, operadores lógicos e instruções de entrada e saída. Durante os encontros foram realizadas demonstrações sobre o funcionamento de dispositivos eletrônicos e robótica, elaboração de algoritmos, construção de robôs (utilizando Arduino) e atividades de lógica de programação utilizando fluxogramas para representação visual de um algoritmo. Após a criação dos fluxogramas, foi utilizado o software Modelix System para transformação automática dos diagramas em linguagem de programação e envio para o Arduino. Os resultados

\footnotetext{
1 A denominação de "conceitos intermediários" é usada neste trabalho para representar conceitos posteriores aos tópicos introdutórios de programação. Mais informações podem ser encontradas na Seção 4.1.
} 
VIII Congresso Brasileiro de Informática na Educação (CBIE 2019)

Anais dos Workshops do VIII Congresso Brasileiro de Informática na Educação (WCBIE 2019)

foram satisfatórios tanto no desenvolvimento das atividades pelos alunos, quanto na interação em grupo. Com respeito às atividades, todas as lógicas de programação foram criadas e, após os testes com os robôs, foram realizados os ajustes necessários no código. Com relação à interação em grupo, observou-se como aspectos positivos: a troca de informações entre os alunos, divisão de tarefas, discussões e questionamentos.

Zanetti e Oliveira (2015) criaram uma oficina, com seis alunos do curso Técnico em Informática integrado ao ensino médio, para desenvolvimento do pensamento computacional trabalhando os conceitos de entrada e saída, estruturas de decisão e repetição. No ambiente de programação foi utilizada a ferramenta visual Scratch 4 Arduino (S4A) e um robô controlado pelo Arduino. Os resultados, baseados na percepção dos alunos, mostraram que o método facilitou a compreensão dos conceitos de programação e a identificação/correção dos erros. Além disso, 50\% dos participantes se sentiram motivados com a aplicação do método.

Cardoso e Antonello (2015) montaram as disciplinas de Algoritmo e Oficina de Criatividade para trabalhar os conceitos básicos de algoritmo com 46 alunos ingressantes do curso de Sistemas de Informação. As atividades desenvolvidas com os alunos envolveram 1) dinâmicas de grupo, para integração dos alunos; (2) programação visual por blocos, para criação de programas utilizando blocos funcionais; (3) uso do Visualg, para desenvolvimento de pseudocódigos; e (4) kits de Arduino ${ }^{2}$, para aplicação dos conceitos de estruturas de decisão e repetição. Os resultados, baseados em relatos dos alunos, mostraram que o Arduino contribuiu para a melhor compreensão e aprendizado dos conceitos de algoritmo.

Rubio et al. (2015) realizaram uma pesquisa com o objetivo de minimizar a diferença de gêneros nos cursos de introdução a computação. O estudo foi realizado com 76 alunos (47 mulheres e 29 homens) do curso de Biologia para ensino de programação no Matlab. Foram desenvolvidos diversos módulos de aprendizagem constituídos por palestras, com demonstrações sobre o uso do Arduino associado a conceitos de programação (vetores, estruturas de decisão e repetição), e sessões em laboratório para que os alunos exercitassem na prática o que aprenderam. Os dados foram coletados por meio de instrumentos para avaliar a percepção do aluno (modelo $\mathrm{TAM}^{3}$ ) e aprendizagem (exames avaliando as habilidades de programação). A partir disso, os resultados mostraram que o uso dos módulos de aprendizagem foi efetivo, dado que nos grupos que utilizaram essa metodologia não houve diferença significativa entre homens e mulheres com respeito à percepção sobre o uso dos conceitos de programação (facilidade, utilidade e intenções futuras) e resultados de aprendizagem.

Apesar do uso do Arduino para o desenvolvimento do raciocínio lógico dos alunos em disciplinas de programação, observa-se que os estudos apresentados nesta seção se concentram no ensino de conceitos básicos (ex.: comandos de entrada/saída, estruturas de decisão/repetição). Nesse sentido, este trabalho visa ampliar as pesquisas

\footnotetext{
${ }^{2}$ Os kits de Arduino foram utilizados apenas na disciplina de Oficina de Criatividade.

${ }^{3}$ O modelo TAM foi utilizado no estudo de Rubio et al. (2015) para avaliar se os alunos possuíam a intenção de programar no futuro.
} 
na área ao investigar o uso do Arduino no ensino de conceitos posteriores aos tópicos introdutórios de uma linguagem de programação (vetor de caractere, estrutura e função).

\section{Método}

O método utilizado neste trabalho baseia-se em um estudo experimental, seguindo o processo de experimentação proposto por Wholin et al. (2012), contemplando as etapas de planejamento, instrumentação, formulação de hipóteses, projeto e execução.

O planejamento consiste na definição do escopo/contexto, objetivo e participantes do estudo. A instrumentação apresenta os materiais utilizados para execução do experimento. As hipóteses são usadas na busca de respostas para uma questão de pesquisa. O projeto experimental detalha as etapas de desenvolvimento do estudo. Por fim, a etapa de execução apresenta e discute os principais resultados. Mais informações sobre cada uma das etapas são apresentadas na próxima seção.

\section{Estudo Experimental}

\subsection{Escopo e Contexto}

A experiência relatada neste trabalho foi realizada no $1^{\circ}$ semestre de 2019, na Universidade Federal de Viçosa - campus Rio Paranaíba, para as turmas da disciplina de Programação ${ }^{4}$. O conteúdo programático da disciplina durante o semestre foi dividido em três partes:

1) Conceitos básicos: estrutura de decisão, estrutura de repetição e vetor/matriz;

2) Conceitos intermediários: vetor de caractere (string), estrutura (struct) e função;

3) Conceitos finais: alocação dinâmica, recursividade e arquivo.

Logo, para este experimento foram selecionados os conteúdos referentes aos conceitos básicos e intermediários para serem trabalhados nas aulas práticas da disciplina de Programação. Essas aulas foram ministradas semanalmente (2 horas/aula) utilizando circuitos Arduino pré-montados. Todas as etapas deste estudo foram realizadas no laboratório de informática e os códigos desenvolvidos na linguagem de programação C.

\subsection{Participantes}

Participaram do experimento duas turmas da disciplina de Programação (T1 e T2) totalizando 62 alunos. Desse conjunto, 12 são mulheres e 50 são homens, sendo 59 alunos do curso de Sistemas de Informação e 3 do curso de Engenharia de Produção. Além disso, 67,74\% alunos são ingressantes, ou seja, estão cursando a disciplina pela primeira vez e 32,26\% são repetentes, ou seja, já cursaram a disciplina anteriormente.

\subsection{Instrumentos}

\subsubsection{Arduino}

\footnotetext{
${ }^{4}$ A disciplina de Programação faz parte da grade dos cursos de Sistemas de Informação e Engenharia de Produção e visa introduzir a linguagem de programação $\mathrm{C}$ e estimular o raciocínio lógico dos alunos.
} 
No presente trabalho foi utilizado a placa Arduino Mega2560, escolhida por ser completa, moderna e contar com aumento significativo na funcionalidade de entrada e saída em relação às outras versões do Arduino [Evans et al. 2013]. Para as aulas práticas foram utilizados 26 kits de Arduino e construídos dois circuitos eletrônicos específicos, ilustrados pela Figura $1^{5}$, para a resolução das listas de exercícios.

O primeiro circuito, à esquerda, utilizou seis LEDs (três vermelhos e três verdes) e seis resistores montados em uma placa de ensaio, sendo cada LED associado a uma porta de saída específica do Arduino. O segundo circuito, à direita, utilizou três resistores montados na placa de ensaio e um LED RGB que consiste em três LEDs encapsulados em um mesmo componente capaz de gerar cores diferentes (vermelho, verde e azul). Cada cor é associada a uma porta de saída específica do Arduino que pode ser controlada individualmente ou combinada para gerar novas cores.
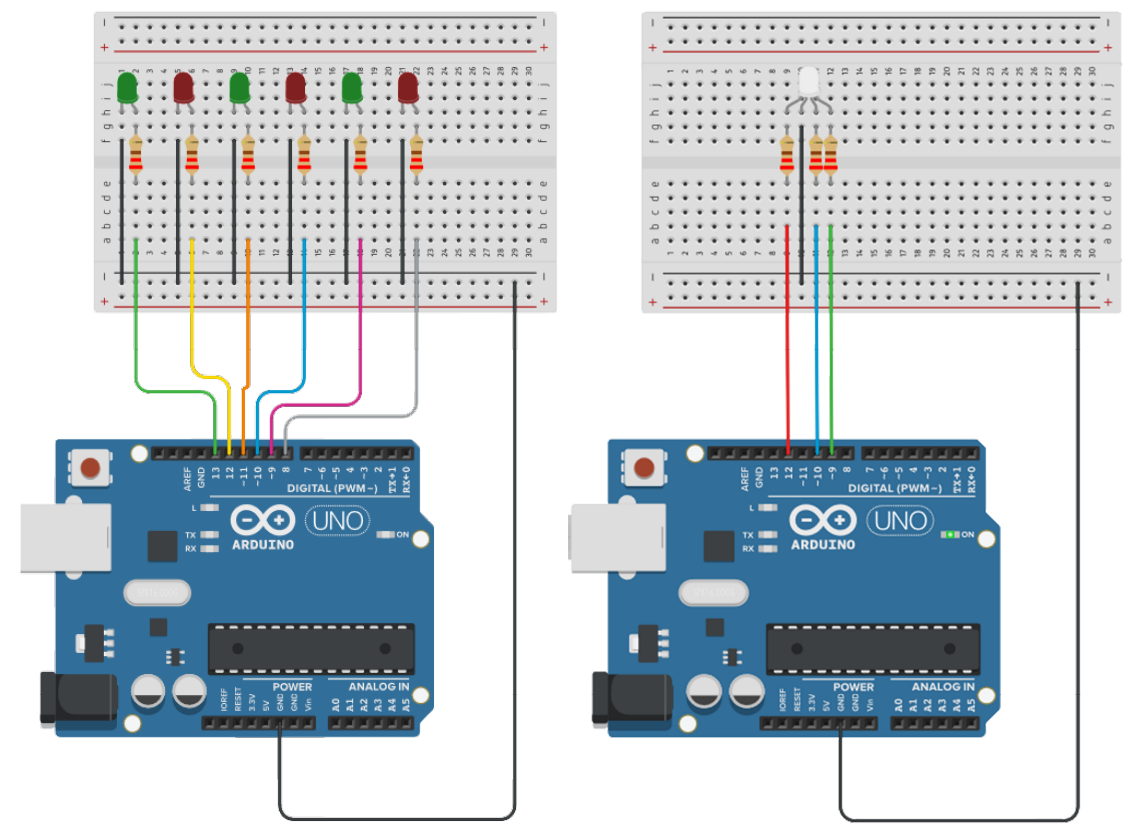

Figura 1. Circuitos eletrônicos criados para as aulas práticas de Programação, à esquerda utilizando seis LEDs e à direita utilizando o LED RGB.

\subsubsection{Listas de Exercícios}

Para cada aula prática foram elaboradas listas com exercícios contextualizados ${ }^{6}$, conforme ilustrado na Figura 2. No total foram produzidas seis listas de exercícios para os conceitos de programação básicos e intermediários, ou seja, uma lista para cada tópico (estrutura de decisão, estrutura de repetição, vetor/matriz, vetor de caractere, estrutura e função). Vale ressaltar que cada lista era formada por quatro exercícios e um

\footnotetext{
5 Os circuitos ilustrados na Figura 1 foram criados pelo simulador Arduino Tinkercad (https://www.tinkercad.com/) que utiliza a placa Arduino Uno.

6 Segundo Freitas et al. (2014), um exercício contextualizado descreve uma situação-problema relacionada ao dia-a-dia do aluno (ex.: cotidiano, Universidade) com o intuito de instigar o raciocínio lógico e capacidade de abstração dos alunos.
} 
VIII Congresso Brasileiro de Informática na Educação (CBIE 2019)

Anais dos Workshops do VIII Congresso Brasileiro de Informática na Educação (WCBIE 2019)

subescalas da motivação interesselaproveitamento, percepção de escolha, percepção de competência e pressão/tensão sejam significativamente diferentes entre o uso do Arduino na resolução de exercícios sobre os conteúdos básicos e intermediários de uma linguagem de programação.

Tabela 1. Hipóteses para cada subescala da motivação.

\begin{tabular}{|c|c|c|}
\hline & Hipótese Nula $\left(\mathrm{H}_{0}\right)$ & Hipótese Alternativa $\left(\mathrm{H}_{1}\right)$ \\
\hline $\begin{array}{l}\text { Interesse e } \\
\text { aproveitamento }\end{array}$ & $\begin{array}{l}\text { Não existe diferença significativa no } \\
\text { interesse/aproveitamento dos alunos } \\
\text { sobre o uso do Arduino para resolução de } \\
\text { exercícios sobre conteúdos básicos e } \\
\text { intermediários de programação. }\end{array}$ & $\begin{array}{l}\text { Existe diferença significativa no } \\
\text { interesse/aproveitamento dos alunos } \\
\text { sobre o uso do Arduino para resolução } \\
\text { de exercícios sobre conteúdos básicos e } \\
\text { intermediários de programação. }\end{array}$ \\
\hline $\begin{array}{l}\text { Percepção de } \\
\text { escolha }\end{array}$ & $\begin{array}{l}\text { Não existe diferença significativa na } \\
\text { percepção de escolha dos alunos sobre o } \\
\text { uso do Arduino para resolução de } \\
\text { exercícios sobre conteúdos básicos e } \\
\text { intermediários de programação. }\end{array}$ & $\begin{array}{l}\text { Existe diferença significativa na } \\
\text { percepção de escolha dos alunos sobre } \\
\text { o uso do Arduino para resolução de } \\
\text { exercícios sobre conteúdos básicos e } \\
\text { intermediários de programação. }\end{array}$ \\
\hline $\begin{array}{l}\text { Percepção de } \\
\text { competência }\end{array}$ & $\begin{array}{l}\text { Não existe diferença significativa na } \\
\text { percepção de competência dos alunos } \\
\text { sobre o uso do Arduino para resolução de } \\
\text { exercícios sobre conteúdos básicos e } \\
\text { intermediários de programação. }\end{array}$ & $\begin{array}{l}\text { Existe diferença significativa na } \\
\text { percepção de competência dos alunos } \\
\text { sobre o uso do Arduino para resolução } \\
\text { de exercícios sobre conteúdos básicos e } \\
\text { intermediários de programação. }\end{array}$ \\
\hline Pressão e tensão & $\begin{array}{l}\text { Não existe diferença significativa na } \\
\text { pressãa } \boldsymbol{e} \text { tensão dos alunos sobre o uso } \\
\text { do Arduino para resolução de exercícios } \\
\text { sobre conteúdos básicos e intermediários } \\
\text { de programação. }\end{array}$ & $\begin{array}{l}\text { Existe diferença significativa na pressãa } \\
\boldsymbol{e} \text { tensão dos alunos sobre o uso do } \\
\text { Arduino para resolução de exercícios } \\
\text { sobre conteúdos básicos e } \\
\text { intermediários de programação. }\end{array}$ \\
\hline
\end{tabular}

\subsection{Etapas do Experimento}

A configuração do experimento é composta por três fases ilustradas pela Figura 3.

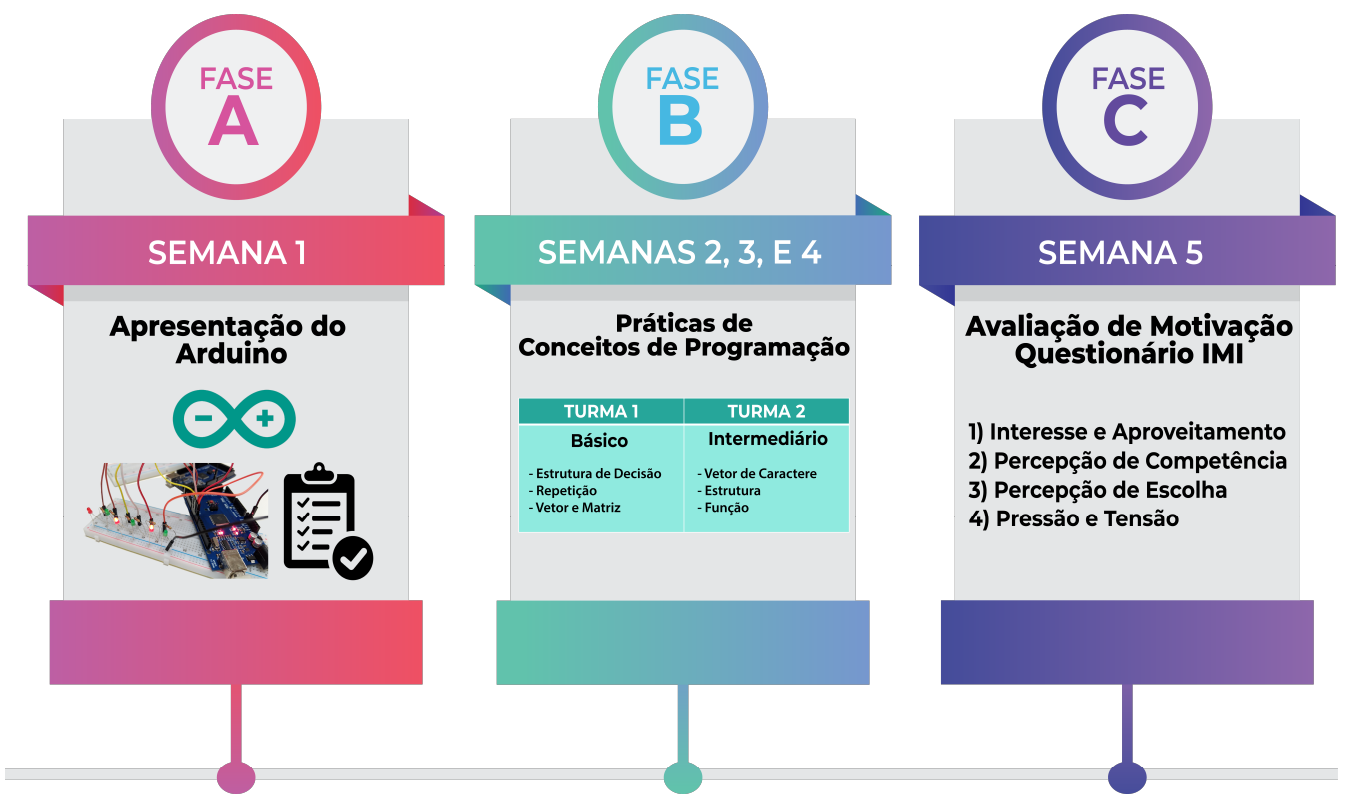

Figura 3. Etapas do experimento. 
Vale ressaltar que as aulas práticas das turmas T1 e T2 ocorreram, respectivamente, nos meses de Abril e Maio de 2019 e que anterior a essas aulas os alunos não tiveram qualquer contato com o Arduino.

\subsubsection{Fase A}

$\mathrm{Na}$ primeira semana dos meses de Abril e Maio, as turmas T1 e T2 receberam, respectivamente, todas as orientações referentes ao Arduino para resolução das listas de exercícios. Em seguida, os alunos foram orientados a resolver um exercício prático para se familiarizar com o editor Arduino.

\subsubsection{Fase B}

Essa etapa foi realizada em três aulas práticas de 2 horas/aula realizadas uma vez por semana. Em cada aula prática foi trabalhado um conteúdo específico:

- Turma 1: semana 2 estrutura de decisão, semana 3 estrutura de repetição e semana 4 vetor/matriz;

- Turma 2: semana 2 vetor de caractere (string), semana 3 estrutura (struct) e semana 4 função.

\subsubsection{Fase C}

Nessa fase as turmas T1 e T2 responderam o questionário IMI avaliando sua motivação em relação às aulas práticas para resolução de exercícios sobre os conteúdos básicos e intermediários de programação.

\section{Resultados e Discussão}

Para análise dos dados foi executado o teste-t com intervalo de confiança de $95 \%$, usando o software SPSS, para comparar a motivação dos grupos referente ao uso do Arduino para a resolução de exercícios sobre os conteúdos básicos e intermediários de programação.

$\mathrm{O}$ resultado do teste- $t$ mostrou que a média da motivação para a subescala percepção de competência para os conceitos intermediários de programação $(\mu=2,97)$ foi significativamente menor $(p<, 05)$ que a média da motivação para os conceitos básicos $(\mu=3,69)$. Isso demonstra que a percepção dos alunos sobre seu desempenho na resolução dos exercícios sobre os conceitos intermediários de programação foi significativamente menor comparado aos alunos que trabalharam nos exercícios sobre os conceitos básicos. Logo, a hipótese nula para essa subescala é negada, ou seja, existe diferença significativa entre o uso da Arduino para o ensino de conceitos básicos e intermediários de programação e, nesse caso, essa diferença foi a favor do ensino dos conceitos básicos. Com respeito às outras subescalas da motivação (interesse/aproveitamento, percepção de escolha, tensão/pressão) a hipótese nula é aceita, ou seja, não houve diferença significativa na motivação dos alunos para essas subescalas com relação ao uso do Arduino na resolução dos exercícios sobre os conceitos básicos e intermediários de programação.

Os resultados encontrados para a subescala da motivação percepção de competência mostram uma visão do aluno pouco explorada na literatura sobre o uso do Arduino no ensino de programação (ex.: <<não me sinto bom, não me saí bem e/ou não me 
VIII Congresso Brasileiro de Informática na Educação (CBIE 2019)

Anais dos Workshops do VIII Congresso Brasileiro de Informática na Educação (WCBIE 2019)

sinto competente [nessa atividade] $>$ ). Acredita-se que esses resultados estejam relacionados à limitação natural do ser humano em processar muitas informações ao mesmo tempo [Sweller et al. 1998], podendo ser a causa de uma sobrecarga cognitiva ${ }^{9}$. Como 74,19\% dos alunos da turma T2 (que trabalhou conceitos intermediários de programação) eram ingressantes (calouros), diversos fatores podem ter contribuído para essa sobrecarga. Por exemplo, falta de familiaridade com o editor de programação Arduino, instruções específicas para execução do código no Arduino, dificuldade de desenvolvimento do raciocínio lógico para resolução dos exercícios, complexidade dos tópicos abordados (vetor de caractere, registro e função), entre outros.

\section{Conclusão}

Esse trabalho realizou um estudo experimental para responder a seguinte questão de pesquisa: "A motivação dos alunos ao utilizar o Arduino na resolução de exercícios sobre conteúdos intermediários de programação é maior do que na resolução de exercícios sobre conteúdos básicos?".

Os resultados, obtidos pelo questionário IMI, mostraram que a percepção de competência dos alunos na resolução de exercícios sobre conceitos intermediários de programação foi significativamente menor do que na resolução de exercícios sobre conceitos básicos. Logo, a resposta à questão de pesquisa formulada para este trabalho é não, dado que os alunos não se sentiram motivados (menos competentes) com o uso do Arduino na resolução de conceitos intermediários de programação.

Como trabalho futuro sugere-se o uso do Arduino em outros contextos de aprendizagem para apoiar o ensino dos conceitos intermediários de programação, por exemplo, na área de Robótica Educacional [Avila e Cavalheiro 2017]. Estudos nessa área enfatizam a importância da inserção gradativa dos conceitos de robótica quando usada para desenvolvimento do raciocínio lógico dos alunos [Silva et al. 2016, Avila e Cavalheiro 2017]. Espera-se com essa abordagem minimizar o problema de sobrecarga cognitiva dos alunos ao utilizar o Arduino.

\section{Referências}

Avila, C., Cavalheiro, S. (2017). "Robótica Educacional como Estratégia de Promoção do Pensamento Computacional - Uma Proposta de Metodologia Baseada em Taxonomias de Aprendizagem". Anais dos Workshops do VI Congresso Brasileiro de Informática na Educação (CBIE), Recife, PE, p. 1192 - 1201.

Banzi, M., Michael S. (2014). Getting started with Arduino: the open source electronics prototyping platform. Make Books - Imprint of: O'Reilly Media, Sebastopol, CA.

Bosse, Y., Gerosa, M. A. (2015). "Reprovações e Trancamentos nas Disciplinas de Introdução à Programação da Universidade de São Paulo: Um Estudo Preliminar". XXIII Workshop sobre Educação em Computação, Recife, PE.

Cardoso, R., Antonello, S. L. (2015). "Interdisciplinaridade, programação visual e robótica educacional: relato de experiência sobre o ensino inicial de programação". Anais dos Workshops do IV CBIE, Maceió, AL, p. 1255-1262.

\footnotetext{
9 A sobrecarga cognitiva ocorre quando o aprendizado de um conteúdo exige do indivíduo diversas habilidades diferentes, sendo que algumas ainda não foram desenvolvidas.
} 
VIII Congresso Brasileiro de Informática na Educação (CBIE 2019)

Anais dos Workshops do VIII Congresso Brasileiro de Informática na Educação (WCBIE 2019)

Deci, E. L., Ryan, R. M. (1985). Intrinsic motivation and self-determination in human behavior. Springer US, $372 \mathrm{p}$.

Evans, M., Noble, J.,Hochenbaum, J. (2013). Arduino in action. Manning Publications, p. 368.

Fernandes, M., Santos, C. A. M. dos, Souza, E. E. P. de, Fonseca, M. G. (2018). "Robótica educacional: Uma ferramenta para ensino de lógica de programação no ensino fundamental", XXIV Workshop de Informática na Escola (WIE), Fortaleza, CE, p. 315-322.

Freitas, M. F., Mota, S., D. S., Soares, L. S., Reis, R. C. D. (2014). "PORTEC: uma ferramenta para auxiliar na abstração dos conceitos de Estrutura de Dados", XXV Simpósio Brasileiro de Informática na Educação (SBIE), Dourados, MS, p. 872-881.

Henrique, M. S., Tedesco, P. C. de A. R. (2017). "Uma Revisão sistemática da Literatura sobre conhecimentos, habilidades, atitudes e competências desejáveis para auxiliar a aprendizagem de programação", Anais dos Workshops do VI Congresso Brasileiro de Informática na Educação (CBIE), Recife, PE, p. 1162-1171.

Pedro, L. Z. (2016). "Uso de Gamificação em Ambientes Virtuais de Aprendizagem para Reduzir o Problema da Externalização de Comportamentos Indesejáveis". Dissertação de Mestrado, Universidade de São Paulo, São Carlos, SP, 152 p.

Queiroz, M. O., Rebouças, A. D. D. S. (2018). "Neurociência e o ensino de programação: Uma revisão sistemática da literatura". XXIX Simpósio Brasileiro de Informática na Educação (SBIE), Fortaleza, CE, p. 1033-1042.

Rubio, M. A., Romero-Zaliz, R., Mañoso, C., Madrid, A. P. de (2015). Closing the gender gap in an introductory programming course. Computers \& Education, 82: 409-420.

Silva, D. P. da, Sidnei, S. S., Jesus, A. M. de, Silva, C. E. P. (2016). "Aplicação de Robótica na Educação de Forma Gradual para o Estímulo do Pensamento Computacional", Anais dos Workshops do V CBIE, Uberlândia, MG, p. 1188-1197.

Sweller, J., Merrienboer, J. J. V., Paas, F. G. (1998). Cognitive architecture and instructional design. Educational psychology review, Springer, v. 10(3): p. 251-296.

Zanetti, H. A. P., Borges, M. A. F., Ricarte, I. L. M. (2016). "Pensamento Computacional no Ensino de Programação: Uma Revisão Sistemática da Literatura Brasileira", XXVII Simpósio Brasileiro de Informática na Educação (SBIE), Uberlândia, MG, p. 21-30.

Zanetti, H. A. P., Oliveira, C. L. V. (2015). "Prática de ensino de Programação de Computadores com Robótica Pedagógica e aplicação de Pensamento Computacional", Anais dos Workshops do IV CBIE, Maceió, AL, p. 1236-1245.

Wohlin, C., Runeson, P., Host, M., Ohlsson, M. C., Regnell, B., Wesslén, A. (2012). Experimentation in Software Engineering. Springer Publishing Company, Incorporated. 